\title{
Increasing $\mathrm{Cu}$ bioavailability inhibits $\mathrm{A} \boldsymbol{\beta}$ oligomers and tau phosphorylation
}

\author{
Peter J. Crouch a,b,c,1, Lin Wai Hunga,c,d,1, Paul A. Adlardc, Mikhalina Cortesc, Varsha Lalc, Gulay Filiz ${ }^{a, b, c}$, \\ Keyla A. Perez ${ }^{a, c, d}$, Milawaty Nurjonoc, Aphrodite Caragounis ${ }^{a, b, c}$, Tai Du ${ }^{a, b, c}$, Katrina Laughton ${ }^{a, c}$, Irene Volitakis ${ }^{a, c}$, \\ Ashley I. Bushc, Qiao-Xin Lia,b,c, Colin L. Mastersc, Roberto Cappaia,c,d, Robert A. Chernyc, Paul S. Donnelly ${ }^{\mathrm{d}, \mathrm{e}}$, \\ Anthony R. White ${ }^{a, b, c, 2,3}$, and Kevin J. Barnham ${ }^{a, c, d, 2,4}$ \\ aDepartment of Pathology, bentre for Neuroscience, eSchool of Chemistry, and dBio21 Molecular Science and Biotechnology Institute, University of
Melbourne, Melbourne, Victorial, 3010, Australia; and 'Mental Health Research Institute of Victoria, Parkville, Victoria 3052, Australia
}

Edited by Peter J. Sadler, University of Warwick, Coventry, United Kingdom, and accepted by the Editorial Board November 21, 2008 (received for review September 11, 2008)

\begin{abstract}
Cognitive decline in Alzheimer's disease (AD) involves pathological accumulation of synaptotoxic amyloid- $\beta(A \beta)$ oligomers and hyperphosphorylated tau. Because recent evidence indicates that glycogen synthase kinase $3 \beta$ (GSK3 $\beta$ ) activity regulates these neurotoxic pathways, we developed an AD therapeutic strategy to target GSK3 $\beta$. The strategy involves the use of copper-bis(thiosemicarbazonoto) complexes to increase intracellular copper bioavailability and inhibit GSK3 $\beta$ through activation of an Akt signaling pathway. Our lead compound Cu"(gtsm) significantly inhibited GSK3 $\beta$ in the brains of APP/PS1 transgenic AD model mice. Cull(gtsm) also decreased the abundance of $A \beta$ trimers and phosphorylated tau, and restored performance of AD mice in the Y-maze test to levels expected for cognitively normal animals. Improvement in the Y-maze correlated directly with decreased A $\boldsymbol{\beta}$ trimer levels. This study demonstrates that increasing intracellular copper bioavailability can restore cognitive function by inhibiting the accumulation of neurotoxic $A \boldsymbol{\beta}$ trimers and phosphorylated tau.
\end{abstract}

Alzheimer's disease | bioinorganic chemistry | glycogen synthase kinase | therapeutic | animal model

A lzheimer's disease (AD) is a neurodegenerative disorder characterized clinically by impaired cognitive performance and pathologically by cerebral deposition of extracellular amyloid plaques and intracellular neurofibrillary tangles. Amyloid plaques in $\mathrm{AD}$ contain aggregated forms of the 39- to 43-aa amyloid- $\beta$ peptide $(\mathrm{A} \beta)$ and $\mathrm{A} \beta$ is strongly implicated as a causative agent responsible for cognitive failure in AD. A diverse range of mechanisms for $A \beta$ toxicity has been reported (1). $A \beta$ is produced from the amyloid precursor protein (APP) (2-5) and readily aggregates to form insoluble, high-molecular-mass amyloid structures. Intermediates on the $\mathrm{A} \beta$ aggregation pathway, primarily low-molecular-mass oligomers such as dimers and trimers, exhibit the greatest neurotoxicity (6-8). In addition to A $\beta$ oligomers, aberrantly phosphorylated microtubuleassociated protein tau is also associated with cognitive decline in AD (9). Intracellular neurofibrillary tangles in the AD brain contain hyperphosphorylated tau, and $\mathrm{A} \beta$ induced cognitive deficits characteristic of AD transgenic mice are attenuated by decreasing levels of endogenous tau (10).

It is now widely recognized that a truly effective therapeutic compound for treating $\mathrm{AD}$ needs to attenuate both the $\mathrm{A} \beta$ - and tau-mediated pathologies. Recent positive outcomes for PBT2 in clinical and preclinical trials are therefore pertinent. Lannfelt et al. (11) demonstrated in phase IIa clinical trials that PBT2 lowers plasma $\mathrm{A} \beta$ levels and attenuates cognitive decline, and Adlard et al. (12) have shown that PBT2 decreases interstitial A $\beta$ and phosphorylated tau in the brains of AD model mice. PBT2 is a secondgeneration $8-\mathrm{OH}$ quinoline, which, unlike its predecessor clioquinol, lacks iodine and was selected for clinical development because of its easier chemical synthesis, higher solubility, and increased blood-brain barrier permeability. Its clinical application was developed based on the potential to inhibit $\mathrm{A} \beta$ toxicity through modulation of $\mathrm{A} \beta$-metal interactions (13-16), but the in vivo mechanism of action for PBT2 still requires elucidation. Cell culture studies have provided mechanism of action data to show that membrane-permeable compounds with the potential to form metal complexes, such as PBT2, can inhibit $\mathrm{A} \beta$ toxicity by increasing cellular metal ion bioavailability and activating neuroprotective cell signaling pathways (17-20). Central to these pathways is inhibition of glycogen synthase kinase $3 \beta$ (GSK $3 \beta)$. Inhibition of this kinase in vitro increases expression of $A \beta$-degrading proteases (20), and GSK $3 \beta$ is a kinase capable of phosphorylating tau $(21,22)$. In this study, we tested the hypothesis that accumulation of $\mathrm{A} \beta$ and phosphorylated tau could be prevented in vivo by inhibiting GSK3 $\beta$ with an agent that increases intracellular bioavailability of copper. To do this we used the bis(thiosemicarbazone) compounds $\mathrm{Cu}^{\mathrm{II}}$ (gtsm) and $\mathrm{Cu}^{\mathrm{II}}$ (atsm) (Fig. 1). We were able to demonstrate that inhibition of GSK $3 \beta$ activity with $\mathrm{Cu}^{\mathrm{II}}(\mathrm{gtsm})$, but not the control compound $\mathrm{Cu}^{\mathrm{II}}(\mathrm{atsm})$, decreased abundance of $\mathrm{A} \beta$ trimers and phosphorylated tau in vivo. Importantly, treatment with $\mathrm{Cu}^{\mathrm{I}}$ $\mathrm{I}(\mathrm{gtsm})$ reversed cognitive deficits in APP/PS1 transgenic AD mice.

\section{Results}

Cu"(gtsm) Inhibits GSK3 $\beta$ and Decreases Tau Phosphorylation in Vitro. $\mathrm{SH}-\mathrm{SY} 5 \mathrm{Y}$ cells were treated with $\mathrm{Cu}^{\mathrm{II}}(\mathrm{gtsm})$ or $\mathrm{Cu}^{\mathrm{II}}$ (atsm) (Fig. 1) or the vehicle control dimethyl sulfoxide (DMSO). These metal complexes were used because both compounds are cell permeable and deliver $\mathrm{Cu}$ into the cell. Importantly, once $\mathrm{Cu}^{\mathrm{II}}(\mathrm{gtsm})$ is exposed to the intracellular reducing environment, $\mathrm{Cu}^{\mathrm{II}}$ is reduced to $\mathrm{Cu}^{\mathrm{I}}$, causing it to dissociate from the ligand and to increase $\mathrm{Cu}$ bioavailability. The negative control $\mathrm{Cu}^{\mathrm{II}}(\mathrm{atsm})$ does not reduce and dissociate under normal intracellular conditions, and therefore does not increase $\mathrm{Cu}$ bioavailability $(18,23-26)$. We found both metal complexes increased cellular $\mathrm{Cu}$ levels several hundredfold

Author contributions: P.J.C., L.W.H., P.A.A., R.A.C., P.S.D., A.R.W., and K.J.B. designed research; P.J.C., L.W.H., P.A.A., M.C., V.L., G.F., K.A.P., M.N., A.C., T.D., K.L., I.V., and P.S.D. performed research; P.J.C., L.W.H., A.I.B., Q.-X.L., C.L.M., R.C., R.A.C., P.S.D., A.R.W., and K.J.B. contributed new reagents/analytic tools; P.J.C., L.W.H., P.A.A., R.A.C., P.S.D., A.R.W. and K.J.B. analyzed data; and P.J.C., L.W.H., P.S.D., A.R.W., and K.J.B. wrote the paper.

The authors declare no conflict of interest.

This article is a PNAS Direct Submission. P.J.S. is a guest editor invited by the Editorial Board. ${ }^{1}$ P.J.C. and L.W.H. contributed equally to this work.

${ }^{2}$ A.R.W. and K.J.B. contributed equally to this work.

${ }^{3}$ To whom correspondence may be addressed at: Level 2, Alan Gilbert Building, 161 Barry Street, University of Melbourne, Victoria 3010, Australia. E-mail: arwhite@unimelb. edu.au.

${ }^{4}$ To whom correspondence may be addressed at: Level 4, Bio21 Institute, University of Melbourne, 30 Flemington Road, Parkville, Victoria 3010, Australia. E-mail: kbarnham@ unimelb.edu.au.

This article contains supporting information online at www.pnas.org/cgi/content/full/ 0809057106/DCSupplemental.

C 2009 by The National Academy of Sciences of the USA 
<smiles>CNC1=NN2C=CC3=NN(CC3)C2[Si]1</smiles><smiles>CNC1=NN2N(C)C(C)=C(C)N2N=C(NC)[C@H](Cl)S1</smiles>

Fig. 1. Structures of Cull(gtsm) [glyoxalbis(N (4)-methylthiosemicarbazonato) Cu"l and Cu"(atsm) [diacetylbis(N (4)-methyl-3-thiosemicarbazonato)Cu"].

(Fig. $2 A, P=0.008$ and $P=0.0002$ ). The data in Fig. $2 A$ are consistent with intracellular accumulation of $\mathrm{Cu}^{\mathrm{I}}$ when cells are treated with $\mathrm{Cu}^{\mathrm{II}}(\mathrm{gtsm})$ and an intracellular accumulation of intact $\mathrm{Cu}^{\mathrm{II}}\left(\right.$ atsm) when treated with $\mathrm{Cu}^{\mathrm{II}}($ atsm) . Western blot analyses revealed $\mathrm{Cu}^{\mathrm{II}}(\mathrm{gtsm})$, but not $\mathrm{Cu}^{\mathrm{II}}($ atsm $)$, induced GSK3 $\beta$ phosphorylation at serine-9 (ser9) via its upstream kinase, protein kinase B (Akt), and that phosphorylation of the associated extracellular signal-related kinase 1/2 (ERK1/2) was also increased (Fig. 2B). These data demonstrate that increased bioavailability of intracellular $\mathrm{Cu}$ rather than elevated cellular $\mathrm{Cu}$ per se is required to induce cellular pathways that lead to increased GSK $3 \beta$ phosphorylation at ser9 (i.e., GSK3 $\beta$ inhibition). We have shown previously that induction of these cellular pathways using metal complexes leads to decreased levels of $A \beta$ in cell cultures because of increased expression of $\mathrm{A} \beta$-degrading proteases $(17,18,20)$.

To determine whether $\mathrm{Cu}^{\mathrm{II}}(\mathrm{gtsm})$-mediated inhibition of GSK $3 \beta$ in SH-SY5Y cells inhibited activity of the kinase toward its substrate tau, we determined levels of tau phosphorylation by Western blot analysis. Tau phosphorylation at ser404 was decreased in $\mathrm{Cu}^{\mathrm{II}}(\mathrm{gtsm})$-treated cells by $64 \%$ (Fig. $2 C, P=0.002$ ), but unaltered in $\mathrm{Cu}^{\mathrm{II}}(\mathrm{atsm})$-treated cells $(P=0.376)$. Phosphorylation of tau at ser396 was also decreased by treating with

AsH-SY5Y Cu content
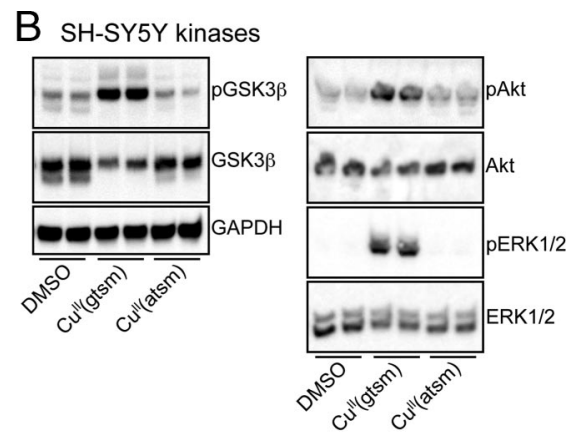

CSH-SY5Y Tau, Western blot and densitometry
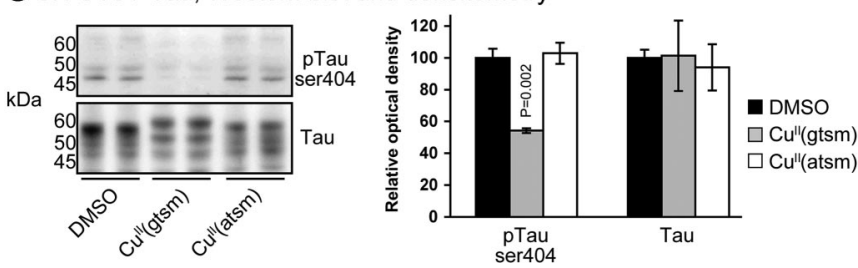

Fig. 2. Cull(gtsm) modulates GSK3 $\beta$ signaling pathways and inhibits tau phosphorylation in SH-SY5Y cells. (A) ICP-MS data showing Cu"(gtsm) and Cu"(atsm) both dramatically increase cellular levels of Cu in SH-SY5Y cells. Cu content of DMSO treated cells was $34 \mathrm{nmol}$ of Cu per milligram of protein. (B) Western blot analyses reveal that Cull(gtsm), but not Cu"(atsm), induced phosphorylation of GSK3 $\beta$, Akt, and ERK1/2. (C) Western blot analysis showing that $\mathrm{Cu}$ "lgtsm) inhibits tau phosphorylation at ser404 without affecting abundance of total tau. $P$ values shown indicate that treatments induced significant effects compared with the DMSO control. Data in A and densitometry data in $C$ are mean values \pm SEM.
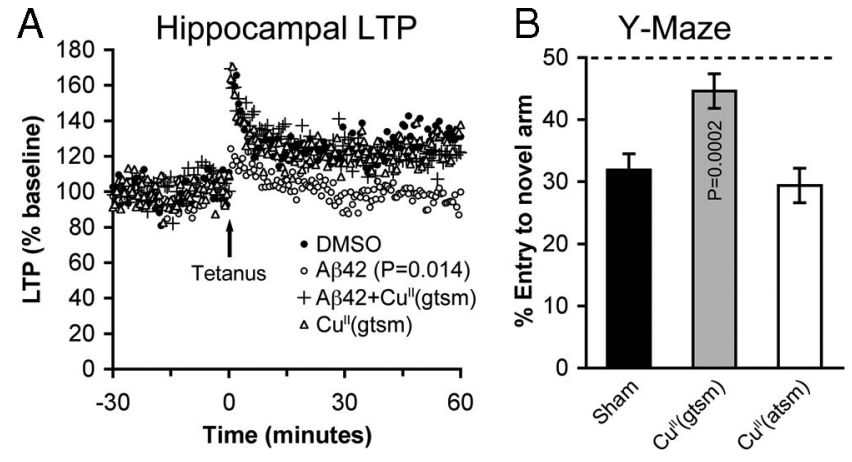

Fig. 3. $C u^{\prime \prime}(g t s m)$ rescues $A \beta$-mediated inhibition of hippocampal long-term potentiation and restores cognitive perfomance of AD mice. $(A)$ LTP field potential recordings demonstrate that compared with DMSO control, synthetic $A \beta 42$ inhibits LTP by $28 \%$, but that the inhibitory potential of $A \beta$ is prevented when hippocampal slices are cotreated with $2 \mu \mathrm{M} \mathrm{Cu}$ "lgtsm). (B) $Y$-maze test for spatial learning and memory shows that untreated AD mice exhibit impaired cognitive performance compared with levels expected for cognitively normal mice (dashed line), and that Cu" (gtsm) restores cognition of AD mice. Cu"(atsm), which unlike Cu"l(gtsm) does not increase cellular $\mathrm{Cu}$ bioavailability, did not improve cognitive performance of AD mice. $P$ values shown indicate that treatments induced significant effects compared with the relevant control. Data in $B$ are mean values \pm SEM.

$\mathrm{Cu}^{\mathrm{II}}(\mathrm{gtsm})$ [supporting information (SI) Fig. S1. The abundance of total tau was unchanged for all treatments (Fig. 2C).

Cu"(gtsm) Rescues $\boldsymbol{A} \boldsymbol{\beta}$-Induced Inhibition of LTP. A $\beta$ has strong] inhibitory potential toward hippocampal long-term potentiation $(\operatorname{LTP})(7,27)$, and metal complex-mediated activation of neuroprotective pathways can decrease $\mathrm{A} \beta$ levels in vitro $(17,18$, 20). Therefore, we determined whether $\mathrm{Cu}^{\mathrm{II}}(\mathrm{gtsm})$ was able to rescue $\mathrm{A} \beta$-induced inhibition of LTP. When included in the artificial cerebrospinal fluid (ACSF) at $2 \mu \mathrm{M}$, synthetic $\mathrm{A} \beta 42$ inhibited LTP in hippocampal slices from wild-type C57/bl6 mice by $28 \%$ (DMSO treatment fEPSP $=130.4 \%, \mathrm{~A} \beta$ treatment fEPSP $=94.5 \%$, Fig. $3 A, P=0.01)$. Including $1 \mu \mathrm{M} \mathrm{Cu}^{\mathrm{II}}(\mathrm{gtsm})$ in the ACSF prevented A $\beta$ toxicity and returned LTP to levels that were not significantly different to controls $\left(\mathrm{A} \beta+\mathrm{Cu}^{\mathrm{II}}(\mathrm{gtsm})\right.$ treatment fEPSP $=121.4 \%, P=0.308) . \mathrm{Cu}^{\mathrm{II}}(\mathrm{gtsm})$ alone had no effect on LTP $\left(\mathrm{Cu}^{\mathrm{II}}(\mathrm{gtsm})\right.$ treatment fEPSP $=127.6 \%$, Fig. $3 \mathrm{~A}$, $P=0.4557)$.

Cu"(gtsm) Improves Cognitive Performance of AD Mice in Y-Maze. Based on our in vitro data showing that $\mathrm{Cu}^{\mathrm{II}}(\mathrm{gtsm})$ activates potentially neuroprotective cell signaling pathways (Fig. 2) and prevents $\mathrm{A} \beta$-mediated inhibition of LTP (Fig. $3 A$ ) we treated APP/PS1 transgenic AD mice with $\mathrm{Cu}^{\mathrm{II}}(\mathrm{gtsm})$ at $10 \mathrm{mg} / \mathrm{kg}$ of body weight $(n=15)$ or vehicle control (sham treatment) $(n=14)$ by daily gavage. Sham- and $\mathrm{Cu}^{\mathrm{II}}(\mathrm{gtsm})$-treated $\mathrm{AD}$ mice were analyzed for cognitive performance by using a 3 -arm radial maze (Y-maze) for spatial working memory and learning (28). Sham-treated AD mice exhibited poor recognition of the Y-maze novel arm (33\% entry to novel arm, Fig. $3 B$ ), demonstrating impaired cognitive performance consistent with previous studies $(29,30)$. By contrast, the $\mathrm{Cu}^{\mathrm{II}}(\mathrm{gtsm})$-treated mice showed significantly improved cognitive performance with $48 \%$ entry to the novel arm (Fig. $3 B, P=$ $0.0002)$. These $\mathrm{Y}$-maze data indicate $\mathrm{Cu}^{\mathrm{II}}(\mathrm{gtsm})$ restored cognitive performance of the $\mathrm{AD}$ mice to levels expected for healthy, cognitively normal mice. In contrast to $\mathrm{Cu}^{\mathrm{II}}(\mathrm{gtsm})$, and consistent with our hypothesis that the therapeutic effects of $\mathrm{Cu}^{\mathrm{II}}(\mathrm{gtsm})$ are based on the compound's capacity to increase intracellular $\mathrm{Cu}$ bioavailability, treating the $\mathrm{AD}$ mice with $\mathrm{Cu}^{\mathrm{II}}(\mathrm{atsm})$ did not affect cognitive performance in the Y-maze test, even at an elevated dose of $100 \mathrm{mg} / \mathrm{kg}$ of body weight (Fig. $3 B, P=0.280$ ). 
A $A D$ mouse brain kinases, Western blot and densitometry
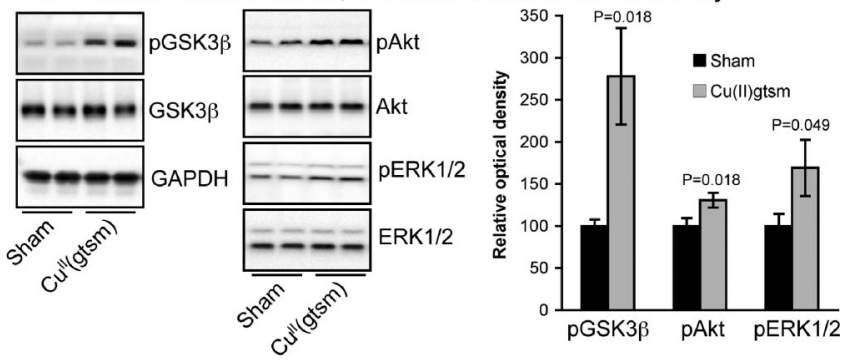

B AD mouse brain Tau, Western blot and densitometry
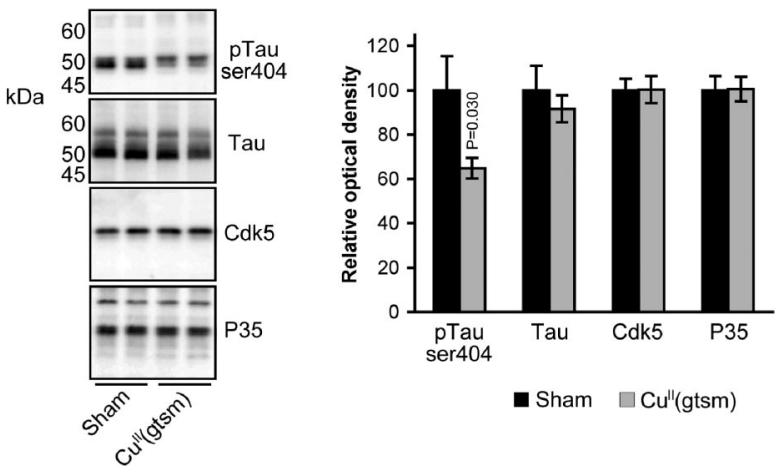

Fig. 4. Cull(gtsm) modulates GSK3 $\beta$ signaling pathways and inhibits tau phosphorylation in the brains of $A D$ mice. $(A)$ Western blot and densitometry analyses showing $\mathrm{Cu}^{\text {Il }}(\mathrm{gtsm})$ increases phosphorylation of GSK3 $\beta$, Akt and ERK $1 / 2$ in brains of AD mice. ERK1/2 could not be detected in PBS extracts of mouse brains and data for ERK $1 / 2$ was therefore obtained by using cell lysis buffer as described in the methods. Data for all other proteins was obtained by using PBS extracts. $(B)$ Western blot and densitometry analysis of brain samples shows tau phosphorylation at ser404 is decreased after treatment with Cull(gtsm) in the absence of effects on total tau, cdk5, or P35. All Western blot densitometry data are normalized for the loading control GAPDH. $P$ values shown indicate that treatments induced significant effects compared with the sham control. Densitometry data in $A$ and $B$ are mean values \pm SEM.

Cu"(gtsm) Inhibits GSK3 $\beta$ in Vivo. Biochemical examination of brain tissue from sham- and $\mathrm{Cu}^{\mathrm{II}}(\mathrm{gtsm})$-treated $\mathrm{AD}$ mice revealed strong consistency with data from experiments using SH-SY5Y cells. $\mathrm{Cu}^{\mathrm{II}}(\mathrm{gtsm})$ induced increased ser9 phosphorylation of GSK $3 \beta$ via its upstream kinase Akt, induced phosphorylation of ERK1/2 (Fig. $4 A, P=0.018,0.018$, and 0.049, respectively). Concomitant with the $\mathrm{Cu}^{\mathrm{II}}(\mathrm{gtsm})$-mediated inhibition of GSK3 $\beta$ was a decrease in detectable levels of phosphorylated tau (Fig. $4 B$, $P=0.030)$. Abundance of total tau was not altered in the $\mathrm{Cu}^{\mathrm{II}}$ (gtsm)-treated mice (Fig. $4 B, P=0.2248$ ).

In addition to GSK $3 \beta$, cyclin-dependent kinase 5 (cdk5) with its regulatory cofactor P35 can also phosphorylate tau (31). We therefore examined whether decreased phosphorylation of tau in AD mice involved decreased cdk5/P35. Neither cdk5 nor P35 levels were altered in the brains of AD mice after treatment with $\mathrm{Cu}^{\mathrm{II}}$ (gtsm) (Fig. $4 B, P=0.49$ and 0.47 , respectively), indicating that decreased tau phosphorylation in vivo is more likely the result of GSK $3 \beta$ inhibition.

Cu"(gtsm) Decreases Brain A $\boldsymbol{\beta}$ Trimer Levels in AD Mice. Because increasing cellular metal ion bioavailability prevents $A \beta$ accumulation in vitro through inhibition of GSK3 $\beta$ (20), we examined whether $\mathrm{Cu}^{\mathrm{II}}$ (gtsm)-mediated inhibition of GSK3 $\beta$ in vivo (Fig. $4 A$ ) affected $\mathrm{A} \beta$ levels in the brains of $\mathrm{AD}$ mice. ELISA analyses demonstrated that $A \beta$ in the PBS soluble fraction of the brain from $\mathrm{Cu}^{\mathrm{II}}$ (gtsm)-treated mice was unaltered, but that $\mathrm{A} \beta$ in the PBSinsoluble fraction was decreased by $24 \%$ (Fig. $5 A, P=0.033$ ). More

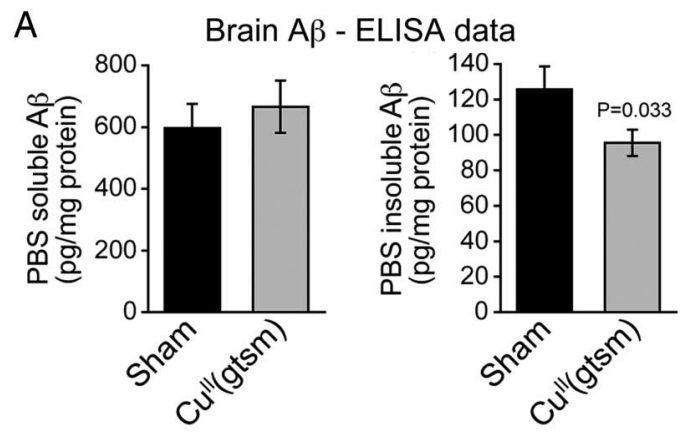

B Brain A $\beta$ - SELDI-TOF MS
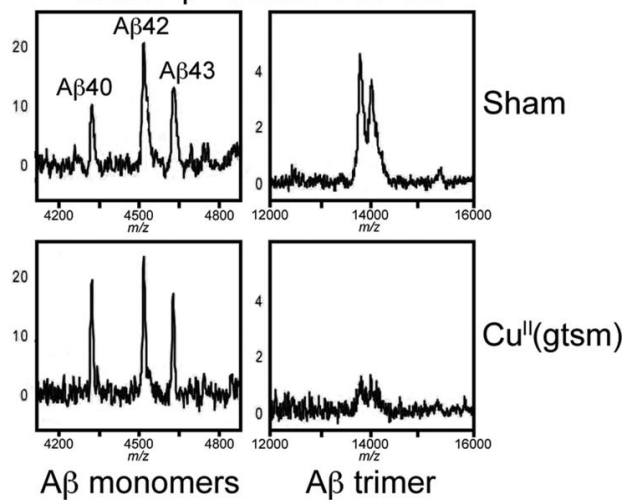

$A \beta$ monomers $\quad A \beta$ trimer

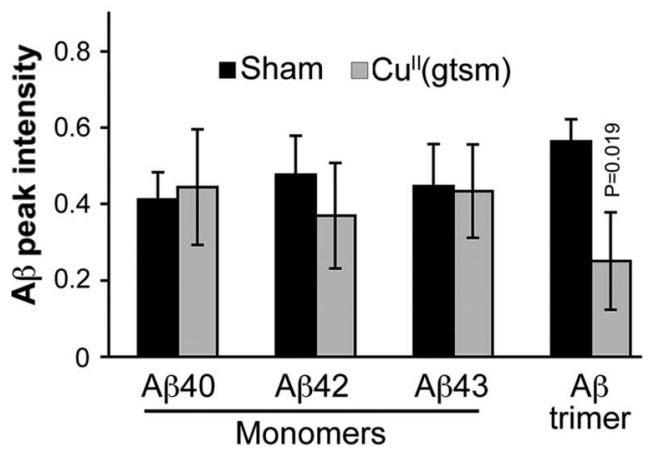

C $A \beta$ trimer and $Y$-maze correlation

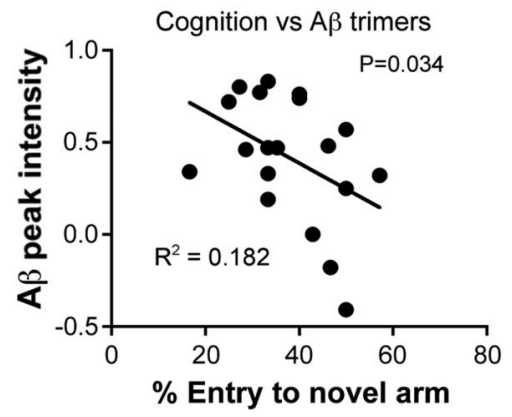

Fig. 5. Cull(gtsm) inhibits accumulation of PBS insoluble $A \beta$ trimers in the brains of AD mice. (A) ELISA data showing that overall levels of PBS insoluble $A \beta$, but not PBS soluble $A \beta$, are decreased in the brains of $\mathrm{Cu}^{\prime \prime}(\mathrm{gtsm})$-treated AD mice. (B) SELDI-TOF MS analysis showing A $\beta$ trimers are decreased in the PBS-insoluble fraction of Cul"(gtsm)-treated mice, but monomer levels are unaffected. All SELDI-TOF MS data are normalized for total brain protein and are expressed as $A \beta$ peak intensity per milligram of protein. (C) Correlation analysis showing cognitive performance in $\mathrm{Y}$-maze testing of $A D$ mice improves with decreasing A $\beta$ trimer levels in the PBS-insoluble fraction of the brain. $P$ values shown indicate that treatments induced significant effects compared with the sham control. Data in $A$ and $A \beta$ peak intensity data in $B$ are mean values \pm SEM. 
rigorous examination of PBS-insoluble brain samples using surface enhanced laser desorption ionization time-of-flight (SELDI-TOF) mass spectrometry (MS) was performed to establish whether the ELISA data were due to an overall decrease in total brain $A \beta$ or due to decreased abundance of specific $\mathrm{A} \beta$ species. The SELDI-TOF MS data show that although monomeric forms of $\mathrm{A} \beta 40, \mathrm{~A} \beta 42$, and $\mathrm{A} \beta 43$ resolved discretely with accurate molecular masses, their levels were not altered by $\mathrm{Cu}^{\mathrm{II}}$ (gtsm) $(P=0.43,0.28$ and 0.47 , respectively). By contrast, a readily detectable $\mathrm{A} \beta$ species with a molecular mass of 13,801 Da, equivalent to an $\mathrm{A} \beta$ trimer, was significantly decreased in $\mathrm{Cu}^{\mathrm{II}}$ (gtsm)-treated mice (Fig. $5 B, P=$ 0.02 ). A mass spectrometry technique has not previously supported the existence of an $A \beta$ trimer in vivo. The second peak shown in Fig. $5 B$ at $14,018 \mathrm{Da}$ is consistent with the $\mathrm{A} \beta$ trimer containing a sinapinic acid adduct; sinapinic acid is part of the SELDI-TOF matrix. Other $A \beta$ oligomer species such as dimers, tetramers, and $\mathrm{A} \beta 56^{*}$ were not detected when using SELDI-TOF MS. Similarly, trimers containing A $\beta 40$ were not detected. Examination via immunohistochemistry revealed treatment with $\mathrm{Cu}^{\mathrm{II}}$ (gtsm) did not affect $\mathrm{A} \beta$ plaque deposition in the brains of AD mice (Fig. S2, $P=0.39$ ).

Although numerous studies demonstrate the neurotoxic potential of $\mathrm{A} \beta$ trimers (32) there is little in vivo evidence to show specific reduction of their abundance in the brain correlates directly with improved cognitive performance. To address this, we performed correlation analyses of brain $A \beta$ trimer levels and performance of mice in the $\mathrm{Y}$-maze test. These analyses revealed that mice with lower levels of trimeric $\mathrm{A} \beta$ in the brain performed better in the Y-maze test $\left(R^{2}=0.18, P=0.034\right)$ (Fig. $\left.5 C\right)$. These data, therefore, provide in vivo evidence for the role of $\mathrm{A} \beta$ trimers in impaired cognitive performance in $\mathrm{AD}$ mice and indicate that therapeutic inhibition of $\mathrm{A} \beta$ trimer accumulation by increasing cellular metal ion bioavailability can rescue impaired cognition.

\section{Discussion}

Therapeutic inhibition of $\mathrm{A} \beta$ accumulation and tau phosphorylation in the brain is a primary target in AD research because aberrant $\mathrm{A} \beta$ accumulation and tau phosphorylation are now widely recognized as the main contributors to the diseaseassociated cognitive decline. Recently a therapeutic compound, PBT2, successfully completed phase IIa clinical trials, and was also shown to decrease $\mathrm{A} \beta$ levels in cerebrospinal fluid and attenuate cognitive decline in $\mathrm{AD}$ patients (11). Importantly, a preclinical study of PBT2 demonstrated that the compound decreased interstitial $A \beta$ as well as phosphorylated tau in the brains of $\mathrm{AD}$ model mice, leading to their improved cognitive performance (12). Based on previous cell culture studies $(18,20)$ it was speculated that the mechanism of action for PBT2 involves sequestration of $\mathrm{Cu}$ and $\mathrm{Zn}$ from extracellular $\mathrm{A} \beta$ aggregates and subsequent PBT2-mediated delivery of the metal ions intracellularly (12). Data to show the mechanism of action through which a PBT2-mediated increase in cellular metal ions can inhibit accumulation of $\mathrm{A} \beta$ and phosphorylated tau are yet to be presented. We used the btsc metal complexes $\mathrm{Cu}^{\mathrm{II}}$ (gtsm) and $\mathrm{Cu}^{\mathrm{II}}$ (atsm) because these complexes can cross the bloodbrain barrier (33), and as described above, they exhibit remarkably different chemical properties that are determined by the substituents attached to the diimine backbone of the ligand and enable controlled regulation of cellular metal ion bioavailability.

The possibility that metal complexes with the capacity to increase intracellular metal bioavailability can activate neuroprotective cell signaling pathways pertinent to $\mathrm{AD}$ is based on cell culture studies using a range of metal complexes. In particular, treating cell cultures with specific $\mathrm{Cu}$ complexes can activate a cell signaling pathway that leads to increased expression of $\mathrm{A} \beta$-degrading proteases and, therefore, decreased $\mathrm{A} \beta$ levels $(18,20)$. The signaling pathway involves phosphorylation of ERK1/2, JNK, and GSK $3 \beta$, and metal complex-mediated phosphorylation of PI3K/Akt because of activation of the epidermal growth factor receptor is an important upstream event $(17-20,34)$. In vivo support for targeting $\mathrm{A} \beta$ and tau in the AD by modulating metal bioavailability comes from a small number of studies. When administered to AD model mice that develop cognitive deficits, clioquinol and PBT2 both decreased the brain $\mathrm{A} \beta$ burden and improved cognitive performance $(12,14)$. Furthermore, PBT2 and clioquinol both provided promising cognition outcomes in clinical trials $(11,16)$. In a related study, AD mice were treated for 7 months with the metal-ligating compound pyrrolidine dithiocarbamate (PDTC), and the treatment was shown to induce phosphorylation of Akt and GSK3 $\beta$, decrease tau phosphorylation, and improve cognitive performance of the mice (35). Surprisingly, however, PDTC did not change $\mathrm{A} \beta$ levels as determined by ELISA. Common to the beneficial effects of clioquinol, PBT2, and PDTC is that the compounds were all administered without metal ions already bound to the ligand. Therefore, if their capacity to inhibit tau phosphorylation and $\mathrm{A} \beta$ accumulation involved the formation of a metal complex, the activity relied on the compounds interacting with metal ions adventitiously in vivo. Such strategies therefore do not rule out the possibility of nonspecific sequestration of metal ions. We believe this is central to our observed effects for $\mathrm{Cu}^{\mathrm{II}}$ (gtsm) on both $\mathrm{A} \beta$ trimers (Fig. 5) and phosphorylated tau (Fig. 4) in the brains of AD mice, because our cell culture work shows metal-free compounds that rely on adventitious binding of metal ions are considerably less potent than metal complexes where the compound is already loaded with $\mathrm{Cu}(18,20)$.

A $\beta$ potently inhibits hippocampal LTP (32), an in vitro assay used to determine the cellular mechanisms of memory and learning. Studies on $\mathrm{A} \beta$-mediated inhibition of LTP indicate $\mathrm{A} \beta$ trimers as the main toxic $\mathrm{A} \beta$ species (7). Here, we have shown that $\mathrm{Cu}^{\mathrm{II}}(\mathrm{gtsm})$ attenuates $\mathrm{A} \beta$-mediated inhibition of LTP. More importantly, we have shown that treatment with $\mathrm{Cu}^{\mathrm{II}}(\mathrm{gtsm})$ decreases the abundance of $\mathrm{A} \beta$ trimers in the brains of $\mathrm{AD}$ mice and that decreased $\mathrm{A} \beta$ trimer levels correlate directly with improved cognitive performance in the Y-maze test. The significance of the data shown in Fig. $5 B$ and $C$ is that mass spectrometry techniques have not previously been used to verify that apparent $\mathrm{A} \beta$ oligomer species seen on a Western blot have a molecular mass consistent with the $\mathrm{A} \beta$ oligomer. Our SELDI-TOF MS data therefore differentiates between $\mathrm{A} \beta$ trimers and APP C-terminal fragments that have the potential to resolve on SDS/PAGE gels in the region expected for $\mathrm{A} \beta$ oligomers. Previous studies have reported an apparent correlation between decreased abundance of the $\mathrm{A} \beta$ species termed $\mathrm{A} \beta 56^{*}$ and improved cognition based on Western blot methods (36), but we propose that more rigorous $\mathrm{A} \beta$ identification techniques are essential before performing correlation analyses (see Table S1).

$\mathrm{Cu}^{\mathrm{II}}(\mathrm{gtsm})$-mediated inhibition of GSK3 $\beta$ is central to our mechanism of action pathway (Fig. 6) and our observed effects for $\mathrm{Cu}^{\mathrm{II}}(\mathrm{gtsm})$ on $\mathrm{A} \beta$ trimers, tau phosphorylation, and cognitive improvement. Originally described for its capacity to phosphorylate glycogen synthase kinase (37), GSK3 $\beta$ is somewhat unique because its capacity to phosphorylate a substrate is inhibited when the kinase itself is phosphorylated at the regulatory site ser9 (38). Phosphorylation of GSK3 $\beta$ therefore inhibits its kinase activity, and the inhibition of GSK $3 \beta$ gained recognition as a potential therapeutic target in $\mathrm{AD}$ once tau was identified as a GSK3 $\beta$ substrate $(21,22)$. Hyperphosphorylated tau is abundant within neurofibrillary tangles of the AD brain, indicating a loss of tau kinase/phosphatase homeostasis. Promoting a substrate-specific increase in phosphatase activity is practically impossible because of relatively poor phosphatasesubstrate specificity; therefore targeting tau kinases is more 


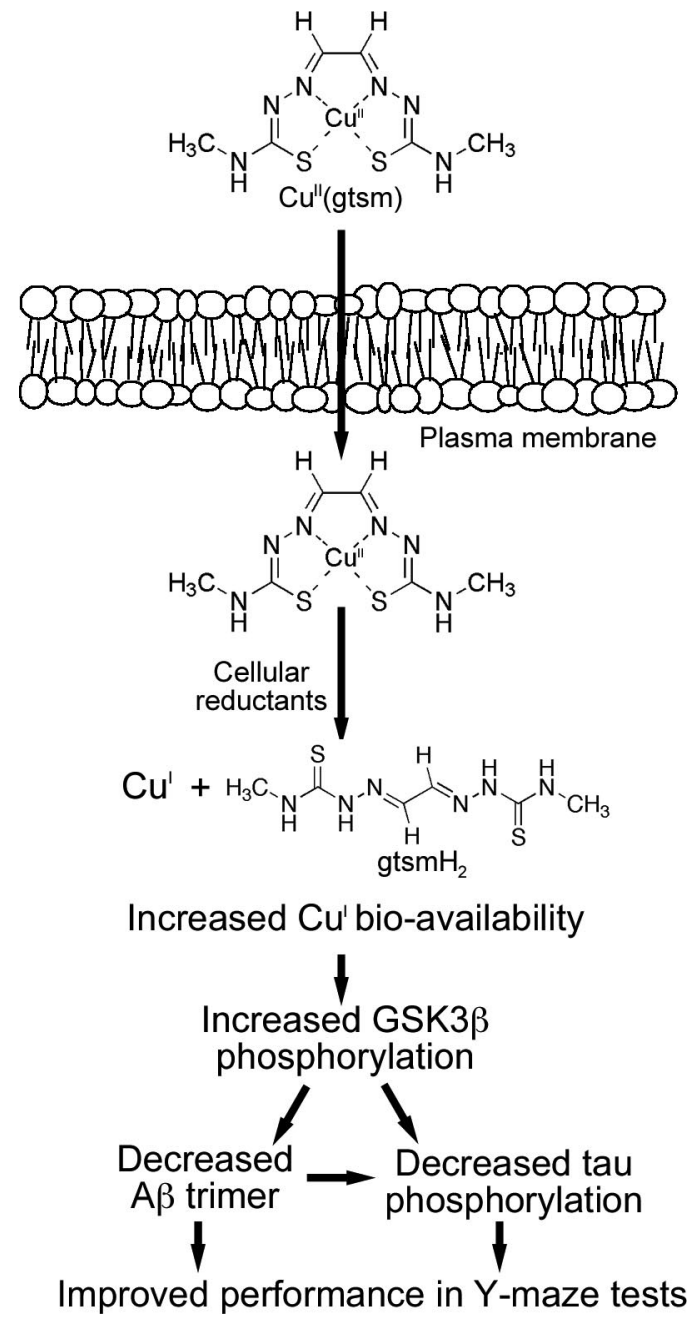

Fig. 6. Schematic overview of proposed mechanism by which Cull(gtsm) decreases $A \beta$ oligomer burden (trimers) and tau phosphorylation in the brains of AD mice. Cell-permeable Cu" (gtsm) dissociates in the presence of intracellular reductants to release $\mathrm{Cu}^{\prime}$ and increase $\mathrm{Cu}$ bioavailability, thereby activating cell signaling pathways that lead to decreased $A \beta$ trimer levels, decreased tau phosphorylation, and improved performance of AD mice in the Y-maze test for spatial learning and memory.

feasible. Here, we have shown that therapeutic inhibition of GSK $3 \beta$ by treating with $\mathrm{Cu}^{\mathrm{II}}$ (gtsm) correlated with decreased tau phosphorylation in vitro and in vivo. Considered together with our observations for decreased $\mathrm{A} \beta$ trimer levels, our data for $\mathrm{Cu}^{\mathrm{II}}(\mathrm{gtsm})$-mediated decreases in tau phosphorylation raise direct questions regarding whether $\mathrm{A} \beta$ trimer levels or aberrantly phosphorylated tau contributes most significantly to effects on cognition. There is conjecture in the literature on this point but growing evidence indicates that although tau is an essential mediator of cognitive decline, $\mathrm{A} \beta$ accumulation is a critical upstream event $(39,40)$. A pertinent study to support this shows that cognitive deficits in an AD mouse model are attenuated by crossing AD mice overexpressing APP with tau ${ }^{-/-}$ mice: $\mathrm{AD} / \mathrm{tau}^{-1-}$ mice exhibit unaltered $\mathrm{A} \beta$ levels compared with $\mathrm{AD} / \mathrm{tau}^{+/+}$mice (10). This work demonstrates a critical role for tau in cognitive decline, but also demonstrates that cognitive deficits are only observed in $\mathrm{AD}$ mice with elevated $\mathrm{A} \beta$ levels. In a more recent study, it was shown that exposure to $\mathrm{A} \beta$ induces tau phosphorylation by promoting GSK $3 \beta$ activity (41). These studies therefore demonstrate an intimate relationship between $\mathrm{A} \beta$ accumulation and tau phosphorylation in the cognitive deficits of $\mathrm{AD}$ and that GSK3$\beta$ activity is an important intermediate. In this study, we have shown that inhibition of GSK $3 \beta$ in vivo by therapeutically increasing $\mathrm{Cu}$ bioavailability decreases $\mathrm{A} \beta$ trimer levels and tau phosphorylation in $\mathrm{AD}$ mice. Importantly, the $\mathrm{Cu}^{\mathrm{II}}(\mathrm{gtsm})$-mediated decrease in $\mathrm{A} \beta$ trimer levels correlated directly with improved cognitive performance in the Y-maze test, and therefore provides strong support for increasing cellular metal ion bioavailability as a valid therapeutic strategy for treating AD.

\section{Materials and Methods \\ The following is a brief summary. Full details are provided in SI Text.}

Cu"(gtsm) and Cu"(atsm). The btsc compounds Cu"(gtsm) and Cu"(atsm) were synthesized as described previously $(42,43)$.

Cell Culture Experiments. SH-SY $5 Y$ cells at $\approx \mathbf{8 0} \%$ confluency were treated with $25 \mu \mathrm{M} \mathrm{Cu}$ "l(gtsm), $25 \mu \mathrm{M} \mathrm{Cu}$ "(atsm) or the vehicle control DMSO in serum-free media for $5 \mathrm{~h}$. Cells collected by centrifugation were resuspended in $50 \mu \mathrm{L}$ of Cytobuster Protein Extraction reagent (Novagen) supplemented with protease and phosphatase inhibitors. Protein content of the cell extracts was determined by using a protein content determination kit (Pierce).

Inductively Coupled Plasma Mass-Spectrometry Determination of Metals Ions. After treating and collecting cells as described above, pelleted cells were rinsed with PBS then analyzed for $\mathrm{Cu}, \mathrm{Fe}, \mathrm{Zn}$, and $\mathrm{Mn}$ by using inductively coupled plasma mass-spectrometry (ICP-MS) as described previously (20).

AD Mice and Treatments. The AD mice used in this study expressed mutant human APP (K670N, M671L) and mutant human PS1 ( $\Delta$ E9) (44). Treatments were administered daily by oral gavage and began when the mice were 5-6 months old. Acid stability studies have shown dissociation of $\mathrm{Cu}$-btsc complexes is unlikely to occur in the gut (45). Mice were tested for cognitive performance by using the $\mathrm{Y}$-maze test, and the brains collected after culling were used for biochemical and immunohistochemical analyses.

Y-Maze Test for Spatial Memory and Learning. Mice were subjected to a 2-trial Y-maze test, with all testing performed during the light phase of the circadian cycle. Three identical arms of the maze were randomly designated start arm novel arm, and other arm. The Y-maze tests were performed after 6 weeks of treatment and consisted of 2 trials separated by a 1-h intertrial interval to assess spatial recognition memory. The first trial (training) was for $10 \mathrm{~min}$, and the mice were allowed to explore only 2 arms (starting arm and other arm). For the second trial (retention), mice were placed back in the maze in the same starting arm, and allowed to explore for 5 min with free access to all 3 arms. By using a ceiling-mounted CCD camera, all trials were analyzed for the number of entries the mice made into each arm. Data are expressed as the percentage of novel arm entries made during the 5-min second trial.

Preparation of Mouse Brain Samples for Biochemical Analyses. Mice were anesthetized, dissected to expose the chest cavity, and then perfused with $\approx 10 \mathrm{~mL}$ of Dulbecco's PBS (Invitrogen) supplemented with heparin, butylated hydroxytolulene, and phosphatase inhibitors. Brains were removed and stored frozen. Right hemispheres were homogenized by sonication in $1 \mathrm{~mL}$ of PBS supplemented with phosphatase inhibitors. Left hemispheres were homogenized mechanically in Protein Extraction Buffer as per SH-SY5Y samples. Soluble and insoluble fractions were separated by centrifugation and protein content determined by using a protein assay kit (Pierce).

Mass Spectrometry Analysis of $\mathbf{A} \boldsymbol{\beta}$ in Brain Tissues. Analysis of $\mathbf{A} \beta$ from brain tissues was performed with surface enhanced laser desorption ionisation time-of-flight (SELDI-TOF) mass spectrometry (MS) using the ProteinChip system (Ciphergen Biosystems). This method enables direct mass-spectrometry identification of individual proteins after immunoaffinity capture purification of the proteins of interest from tissue samples. PBS-soluble and -insoluble extracts of $A D$ mouse brains were subjected to antibody capture using the WO2 antibody for human $A \beta$ (epitope $=A \beta$ residues $5-8$ ), and the mass of each $A \beta$ species was determined by using the ProteinChip reader. Data generated by the ProteinChip software was analyzed by using Biomarker Patterns Software for differential peak intensities of each peptide. All SELDI-TOF MS brain $A \beta$ data are expressed as log-normalized $A \beta$ peak intensity per milligram of brain protein. 
SDS/PAGE and Western Blot Analyses. SH-SY5Y and mouse brain samples were diluted with appropriate homogenization buffer (PBS or cell lysis buffer) and gel loading buffer then heated at $100^{\circ} \mathrm{C}$ for $5 \mathrm{~min}$. Samples were loaded onto glycine gels, proteins electrophoresed at $125 \mathrm{~V}$ for $2-2.5 \mathrm{~h}$, and then transferred onto PVDF membranes (Roche) at $25 \mathrm{~V}$ for $2 \mathrm{~h}$. Membranes were incubated with primary antibody overnight at $4{ }^{\circ} \mathrm{C}$ and then with secondary antibody for 2-3 h. Chemiluminescence images were saved as TIFF files and relative abundance of proteins determined by using NIH ImageJ 1.38x software. All Western blot densitometry data are expressed relative to levels of the loading control glyceraldehyde 3-phosphate dehydrogenase (GAPDH).

Hippocampal LTP. Fourteen- to 40-day-old C57BI6 mice were decapitated under anesthesia. Brains were removed and chilled in ice-cold artificial cerebrospinal fluid (ACSF) and then hippocampal slices prepared and field potential recordings made as described previously (12). LTP was quantified by averaging the data for each slice from 55 to $60 \mathrm{~min}$ after tetanus. Results are presented as mean values ( \pm SEM). When used, $A \beta_{1-42}$ and $C u^{\prime \prime}(\mathrm{gtsm})$ were

1. Crouch PJ, et al. (2008) Mechanisms of $A \beta$ mediated neurodegeneration in Alzheimer's disease. Int J Biochem Cell Biol 40:181-198.

2. Borchelt DR, et al. (1996) Familial Alzheimer's disease-linked presenilin 1 variants elevate $A \beta 1-42 / 1-40$ ratio in vitro and in vivo. Neuron 17:1005-1013.

3. De Strooper B (2003) Aph-1, Pen-2, and nicastrin with nresenilin generate an active $\gamma$-secretase complex. Neuron 38:9-12.

4. Scheuner D, et al. (1996) Secreted amyloid $\beta$-protein similar to that in the senile plaques of Alzheimer's disease is increased in vivo by the presenilin 1 and 2 and APP mutations linked to familial Alzheimer's disease. Nat Med 2:864-870.

5. Vassar R, et al. (1999) Beta-secretase cleavage of Alzheimer's amyloid precursor protein by the transmembrane aspartic protease BACE. Science 286:735-741.

6. Crouch PJ, et al. (2005) Copper-dependent inhibition of human cytochrome coxidase by a dimeric conformer of amyloid- $\beta_{1-42}$. J Neurosci 25:672-679.

7. Walsh DM, et al. (2002) Naturally secreted oligomers of amyloid- $\beta$ protein potently inhibit hippocampal long-term potentiation in vivo. Nature 416:535-539.

8. Shankar GM, et al. (2008) Amyloid- $\beta$ protein dimers isolated directly from Alzheimer's brains impair synaptic plasticity and memory. Nat Med 14:837-842.

9. Ballatore C, Lee VM, Trojanowski JQ (2007) Tau-mediated neurodegeneration in Alzheimer's disease and related disorders. Nat Rev Neurosci 8:663-672.

10. Roberson ED, et al. (2007) Reducing endogenous tau ameliorates amyloid $\beta$-induced deficits in an Alzheimer's disease mouse model. Science 316:750-754.

11. Lannfelt L, et al. (2008) Safety, efficacy, and biomarker findings of PBT2 in targeting A $\beta$ as a modifying therapy for Alzheimer's disease: A phase lla, double-blind, randomised, placebo-controlled trial. Lancet Neurol 7:779-786.

12. Adlard PA, et al. (2008) Rapid restoration of cognition in Alzheimer's transgenic mice with 8-hydroxy quinoline analogs is associated with decreased interstitial A $\beta$. Neuron 59:43-55.

13. Barnham KJ, et al. (2004) Tyrosine gated electron transfer is key to the toxic mechanism of Alzheimer's disease $\beta$-amyloid. FASEB J 18:1427-1429.

14. Cherny RA, et al. (2001) Treatment with a copper-zinc chelator markedly and rapidly inhibits $\beta$-amyloid accumulation in Alzheimer's disease transgenic mice. Neuron 30:665-676.

15. Sampson E, Jenagaratnam L, McShane R (2008) Metal protein attenuating compounds for the treatment of Alzheimer's disease. Coch Database Sys Rev: 1CD005380.

16. Ritchie CW, et al. (2003) Metal-protein attenuation with iodochlorhydroxyquin (clioquinol) targeting $A \beta$ amyloid deposition and toxicity in Alzheimer disease: $A$ pilot phase 2 clinical trial. Arch Neurol 60:1685-1691.

17. Caragounis A, et al. (2007) Differential modulation of Alzheimer's disease amyloid- $\beta$ peptide accumulation by diverse classes of metal ligands. Biochem J 407:435-450.

18. Donnelly PS, et al. (2008) Selective intracellular release of copper and zinc ions from bis(thiosemicarbazonato) complexes reduces levels of Alzheimer disease amyloid- $\beta$ peptide. J Biol Chem 283:4568-4577.

19. Price KA, et al. (2008) Activation of epidermal growth factor receptor by metal-ligand complexes decreases levels of extracellular amyloid- $\beta$ peptide. Int J Biochem Cell Biol 40:1901-1917.

20. White AR, et al. (2006) Degradation of the Alzheimer disease amyloid $\beta$-peptide by metal-dependent up-regulation of metalloprotease activity. J Biol Chem 281:1767017680.

21. Hanger DP, Hughes K, Woodgett JR, Brion JP, Anderton BH (1992) Glycogen synthase kinase-3 induces Alzheimer's disease-like phosphorylation of tau: Generation of paired helical filament epitopes and neuronal localisation of the kinase. Neurosci Lett 147:58-62.

22. Mandelkow EM, et al. (1992) Glycogen synthase kinase-3 and the Alzheimer-like state of microtubule-associated protein tau. FEBS Lett 314:315-321.

23. Xiao Z, Donnelly PS, Zimmermann M, Wedd AG (2008) Transfer of copper between bis(thiosemicarbazone) ligands and intracellular copper-binding proteins: Insights into mechanisms of copper uptake and hypoxia selectivity. Inorg Chem 47:4338-4347. added to the ACSF $30 \mathrm{~min}$ before commencing electrophysiological recordings.

ELISA for $\mathbf{A} \boldsymbol{\beta}$ Detection. $\mathrm{A} \beta$ content of brain samples was determined by using the DELFIA Double Capture ELISA as described previously (20).

Immunohistochemical Quantitation of Mouse Brain A $\boldsymbol{\beta}$ Plaques. Left hemispheres (minus cerebellum) freshly removed from AD mice after 8 weeks treatment were fixed in $10 \%$ (vol/vol) Neutral Buffered Formalin. Histochemical analysis of brain amyloid plaques was performed as described previously by using the anti-A $\beta$ antibody $1 \mathrm{E} 8$ (14).

ACKNOWLEDGMENTS. This work was supported by the National Health and Medical Research Council (NHMRC Program Grant), the Australian Research Council, the ANZ Charitable Trusts (Wicking Trust), and the University of Melbourne (Early Career Researcher Grant Scheme). K.J.B., A.R.W., R.C., and P.A. are NHMRC Fellows. A.I.B. is a current Australian Research Council Fellow. Dr. Giuseppe Ciccotosto kindly provided text for the LTP materials and methods.

24. Dearling JL, Lewis JS, McCarthy DW, Welch MJ, Blower PJ (1998) Redox-active metal complexes for imaging hypoxic tissues: Structure activity relationships in copper(II) bis(thiosemicarbazone) complexes. J Chem Soc Chem Commun 2531-2532.

25. Fujibayashi Y, et al. (1997) Copper-62-ATSM: A new hypoxia imaging agent with high membrane permeability and low redox potential. J Nucl Med 38:1155-1160.

26. Maurer Rl, et al. (2002) Studies on the mechanism of hypoxic selectivity in copper bis(thiosemicarbazone) radiopharmaceuticals. J Med Chem 45:1420-1431.

27. Wang H-W, et al. (2002) Soluble oligomers of $\beta$ amyloid (1-42) inhibit long-term potentiation but not long-term depression in rat dentate gyrus. Brain Res 924:133-140.

28. Olton DS, Samuelson RJ (1976) Remembrance of places past-Spatial memory in rats. $J$ Exp Psychol Anim Behav Process 2:97-116.

29. Arendash GW, et al.(2001) Progressive, age-related behavioral impairments in transgenic mice carrying both mutant amyloid precursor protein and presenilin-1 transgenes. Brain Res 891:42-53.

30. Gordon MN, et al. (2001) Correlation between cognitive deficits and $A \beta$ deposits in transgenic APP+PS1 mice. Neurobiol Aging 22:377-385.

31. Lew J, et al. (1994) A brain-specific activator of cyclin-dependent kinase 5. Nature $371: 423-426$.

32. Selkoe DJ (2008) Soluble oligomers of the amyloid $\beta$-protein impair synaptic plasticity and behavior. Behav Brain Res 192:106-113.

33. Green MA, Klippenstein DL, Tennison JR (1988) Copper(II) bis(thiosemicarbazone) complexes as potential tracers for evaluation of cerebral and myocardial blood flow with PET. J Nucl Med 29:1549-1557.

34. Filiz G, et al. (2008) Clioquinol inhibits peroxide-mediated toxicity through upregulation of phosphoinositol-3-kinase and inhibition of p53 activity. Int J Biochem Cell Biol 40:1030-1042.

35. Malm TM, et al. (2007) Pyrrolidine dithiocarbamate activates Akt and improves spatial learning in APP/PS1 mice without affecting $\beta$-amyloid burden. J Neurosci 27:3712 3721.

36. Mouri $A$, et al. (2007) Oral vaccination with a viral vector containing $A \beta$ CDNA attenuates age-related $A \beta$ accumulation and memory deficits without causing inflammation in a mouse Alzheimer model. FASEB J 21:2135-2148.

37. Embi N, Rylatt DB, Cohen $P(1980)$ Glycogen synthase kinase-3 from rabbit skeletal muscle: Separation from cyclic-AMP-dependent protein kinase and phosphorylase kinase. Eur J Biochem 107:519-527.

38. Sutherland C, Leighton IA, Cohen P (1993) Inactivation of glycogen synthase kinase-3 $\beta$ by phosphorylation: New kinase connections in insulin and growth-factor signalling Biochem J 296:15-19.

39. Gotz J, Chen F, van Dorpe J, Nitsch RM (2001) Formation of neurofibrillary tangles in P301l tau transgenic mice induced by A $\beta 42$ fibrils. Science 293:1491-1495.

40. Lewis J, et al. (2001) Enhanced neurofibrillary degeneration in transgenic mice expressing mutant tau and APP. Science 293:1487-1491.

41. Hu M, Waring JF, Gopalakrishnan M, Li J (2008) Role of GSK-3 $\beta$ activation and $\alpha 7$ $\mathrm{nAChRs}$ in $\mathrm{A} \beta(1-42)$-induced tau phosphorylation in PC12 cells. J Neurochem 106:1371-1377

42. Blower PJ, et al. (2003) Structural trends in copper(II) bis(thiosemicarbazone) radiopharmaceuticals. Dalton Trans 4416-4425.

43. Gingras BA, Suprunchuk T, Bayley CH (1962) The preparation of some thiosemicarbazones and their copper complexes: Part III. Can J Chem 40:1053-1059.

44. Jankowsky JL, et al. (2001) Co-expression of multiple transgenes in mouse CNS: A comparison of strategies. Biomol Engineer 17:157-165.

45. Petering DH (1972) The reaction of 3-ethoxy-2-oxobutyraldehyde bis(thiosemicarbazonato) copper(II) with thiols. Bioinorg Chem 1:273-288. 